\title{
Phenomenon of resistance to Augmentin associated with sensitivity to ampicillin: occurrence and explanation
}

\author{
W BRUMFITT, JMT HAMILTON-MILLER, SHIRLEY DIXSON, RA GARGAN, \\ ANN GOODING
}

From the Department of Medical Microbiology, Royal Free Hospital and School of Medicine, Pond Street, London NW3 $2 Q G$

SUMMARY Ampicillin was found to be some tenfold more active than amoxycillin against Enterobacter cloacae. This finding explains the observation that some Ent cloacae strains are sensitive to ampicillin in the disc test but resistant to Augmentin. Ampicillin was also found to be more active than amoxycillin against Citrobacter freundii and Serratia marcescens. In view of these findings, the practice of using ampicillin discs to predict sensitivity to amoxycillin should be reconsidered. The use of both ampicillin and amoxycillin discs is appropriate if errors are to be avoided.

Amoxycillin has been shown to be at least as active in vitro as ampicillin against many pathogenic bacteria ${ }^{1}$ with notable exceptions, such as Haemophilus infuenzae. Its bactericidal action in vitro and its effectiveness in vivo seems to be superior to that of ampicillin. ${ }^{23}$ Further, amoxycillin is absorbed by mouth almost twice as well as ampicillin. ${ }^{1}$ Because of these properties, amoxycillin can be given in a smaller dose and less frequently than ampicillin, and it now enjoys wide clinical use.

In the laboratory, it is recommended both in the UK and USA that sensitivity testing to amoxycillin be carried out using ampicillin discs. ${ }^{45}$ This is an example of what Barry and Thornsberry ${ }^{4}$ call "class" sensitivity testing, similar to that done in the USA by using a disc of cephalothin to determine sensitivity to the early cephalosporins (such as cephaloridine and cefazolin). The practice of "class" testing, however, may give highly misleading results if incorrectly applied. We have recently made some observations which cast serious doubts on the validity of "class" testing using an ampicillin disc. This investigation started when we isolated several strains of Enterobacter cloacae which appeared sensitive to ampicillin but resistant to Augmentin (amoxycillin + clavulanate) by disc testing (Figure). We briefly drew attention to this phenomenon, in a letter to the Lancet $^{6}{ }^{6}$ and in this report we describe our findings in more detail.

Accepted for publication 26 January 1983

\section{Material and methods}

\section{ANTIBIOTICS}

Ampicillin trihydrate and amoxycillin trihydrate were Laboratory Reference Standards nos 29 and 12, respectively, from Beecham Research Laboratories. Sodium ampicillin was obtained from the hospital pharmacy. Sodium amoxycillin and potassium clavulanate were generously supplied by Beecham Pharmaceuticals, Worthing.

\section{BACTERIAL STRAINS}

Twenty-nine strains of Enterobacter cloacae, 21 Citrobacter freundii and 13 Serratia marcescens were investigated. Four of the Ent cloacae strains had been isolated from patients who had recently received Augmentin, while all the other organisms were from our laboratory collection, which consists of bacteria freshly isolated from clinical material. Identification was by the API 20E system.

\section{DISC SENSITIVITY TESTING}

Augmentin discs (containing $20 \mu \mathrm{g}$ amoxycillin plus $10 \mu \mathrm{g}$ clavulanic acid) were obtained from Oxoid. We made discs containing $20 \mu \mathrm{g}$ ampicillin, $20 \mu \mathrm{g}$ amoxycillin and $20 \mu \mathrm{g}$ ampicillin plus $10 \mu \mathrm{g}$ clavulanic acid. This was done by impregnating Whatman AA discs with the appropriate dilutions of antibiotic.

Plates of Iso-Sensitest agar were flooded with a 1/100 dilution of overnight culture of the test organism. Plates were dried and then one of each of the 


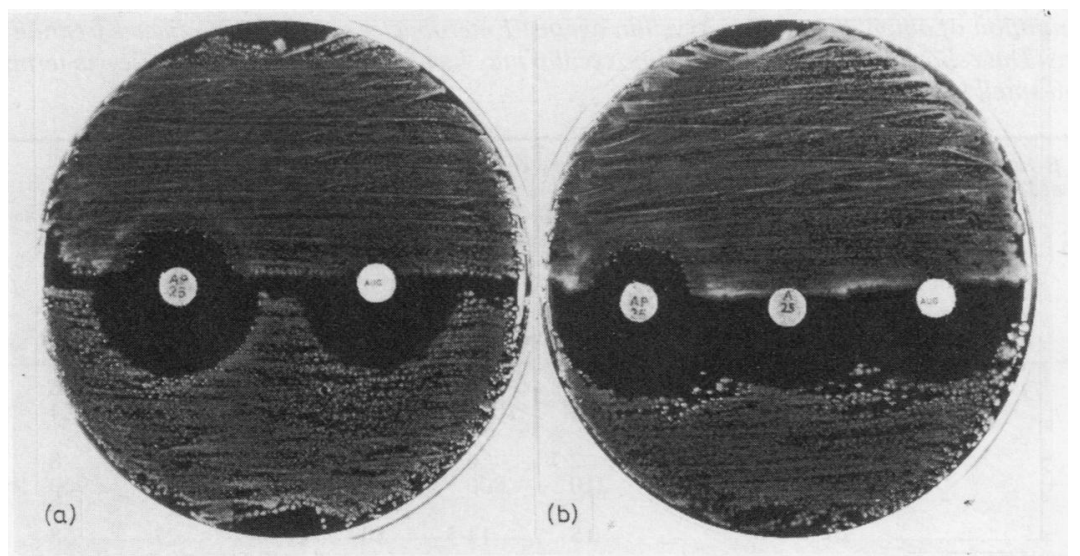

Both plates show results of disc testing with an Enterobacter cloacae strain (top half of each plate). The bottom part of each plate was inoculated with a sensitive control strain of Escherichia coli. AP25 = ampicillin $25 \mu \mathrm{g} ;$ A25 = amoxycillin $25 \mu \mathrm{g} ; \mathrm{AUG}$ $=$ amoxycillin $20 \mu \mathrm{g}+$ clavulanic acid $10 \mu \mathrm{g}$. The results shown in left hand plate are confusing if ampicillin is used as a "class" test (see text). This apparently paradoxical result is explained by the result shown in the right hand plate, namely that the strain is sensitive to ampicillin but resistant to amoxycillin and Augmentin.

discs described above was placed on the surface. Plates were incubated overnight at $37^{\circ} \mathrm{C}$ and zone diameters measured with a millimetre rule. When zone sizes were being compared, a difference of 5 $\mathrm{mm}$ or more was regarded as being significant.

\section{MIC DETERMINATIONS}

These were made by a plate doubling dilution method with Iso-Sensitest agar. Inoculum sizes of $10^{6}$ and $10^{3}$ bacteria were applied to plates by means of a multipoint inoculating device (Denley); plates were read after overnight incubation.

\section{DETECTION OF DESTRUCTION OF AMOXYCILLIN} Organisms were grown in a nutrient broth $(3 \mathrm{ml})$ overnight at $37^{\circ} \mathrm{C} ; 3 \mathrm{ml}$ of a solution of amoxycillin (6 $\mathrm{mg} / \mathrm{ml}$ in sodium phosphate buffer $\mathrm{pH} 8$ ) was added and the mixture incubated at $37^{\circ} \mathrm{C}$ for $6 \mathrm{~h}$. The amount of amoxycillin remaining was then assayed by the hydroxylamine method. ${ }^{7}$ It was found that strains either destroyed all the amoxycillin, or none at all. Strains which destroyed amoxycillin under these conditions are called "penicillinaseproducing" strains.

\section{Results}

As stated earlier, we first observed the apparently paradoxical phenomenon of an organism being ampicillin-sensitive but Augmentin-resistant when testing Ent cloacae. We therefore screened other bacterial species for further evidence of the phenomenon. It was detected only in $C$ freundii and $S$ marcescens, in both of which species it was unusual.

Twenty-one $C$ freundii strains were examined. They fell naturally into two populations:

(a) 14 strains were sensitive to ampicillin, amoxycillin and Augmentin by the disc test. Ampicillin was slightly more active (two to threefold) than amoxycillin against these strains (Table). None of the 14 strains was a penicillinase producer, and clavulanate did not enhance the activity of either ampicillin or amoxycillin. Ampicillin was significantly more active than Augmentin in the disc test against three strains. (b) the remaining seven strains were resistant to ampicillin and to amoxycillin, but sensitive to Augmentin, in the disc test. MICs for these strains were about 100 -fold higher that for sensitive strains (Table); ampicillin was again more active than amoxycillin. All seven strains produced penicillinase.

All $13 S$ marcescens strains showed rather small zones around the ampicillin, amoxycillin and Augmentin discs. Results of MIC determinations indicated that, overall, ampicillin was slightly more active (at most, twofold) than amoxycillin (Table). None of the strains produced penicillinase, and clavulanate did not enhance the activity of either ampicillin or amoxycillin. In four strains ampicillin was significantly more active than Augmentin.

Twenty-nine strains of Ent cloacae fell into two groups.

(a) 24 strains did not produce penicillinase; all were 
Inhibitory concentration of ampicillin and amoxycillin against Enterobacter cloacae, Citrobacter freundii and Serratia marcescens strains. Data show that ampicillin and amoxycillin may have greatly differing activities, in terms of I50 and 190 , for both large and small inocula.

\begin{tabular}{|c|c|c|c|c|c|c|c|c|c|c|}
\hline \multirow[t]{4}{*}{ Bacterial species } & \multirow{4}{*}{$\begin{array}{l}\beta \text {-Lactamase } \\
\text { activity }\end{array}$} & \multirow{4}{*}{$\begin{array}{l}\text { No of } \\
\text { strains }\end{array}$} & \multicolumn{8}{|c|}{ Inhibitory concentrations $(\mu \mathrm{g} / \mathrm{ml})$} \\
\hline & & & \multicolumn{4}{|c|}{$10^{3}$ organisms } & \multicolumn{4}{|c|}{$10^{6}$ organisms } \\
\hline & & & \multicolumn{2}{|l|}{150} & \multicolumn{2}{|l|}{190} & \multicolumn{2}{|l|}{150} & \multicolumn{2}{|l|}{190} \\
\hline & & & $A M P$ & $A M O X$ & $A M P$ & AMOX & $A M P$ & $A M O X$ & $A M P$ & $A M O X$ \\
\hline Ent cloacae & $\overline{+}$ & $\begin{array}{r}24 \\
5\end{array}$ & $\begin{array}{r}7 \\
>1024\end{array}$ & $\begin{array}{r}80 \\
>1024\end{array}$ & $\begin{array}{r}30 \\
>1024\end{array}$ & $\begin{array}{r}410 \\
>1024\end{array}$ & $>1024$ & $\begin{array}{r}128 \\
>1024\end{array}$ & $\begin{array}{r}52 \\
>1024\end{array}$ & $\begin{array}{r}530 \\
>1024\end{array}$ \\
\hline$C$ freundii & $\overline{+}$ & $\begin{array}{r}14 \\
7\end{array}$ & $210^{2 \cdot 7}$ & $800^{6 \cdot 4}$ & $>1024^{7 \cdot 6}$ & $\begin{array}{r}15 \\
>1024\end{array}$ & $330^{6 \cdot 6}$ & $\begin{array}{r}8 \\
900\end{array}$ & $\begin{array}{r}15 \\
>1024\end{array}$ & $\begin{array}{r}60 \\
>1024\end{array}$ \\
\hline$S$ marcescens & - & 13 & 15 & $11 \cdot 5$ & 50 & 70 & 27 & 58 & 100 & 120 \\
\hline
\end{tabular}

* I50 and I90 = inhibitory concentrations of $50 \%$ and $90 \%$, respectively, of strains tested.

$\dagger$ Ability to destroy amoxycillin (see methods).

sensitive to ampicillin by the disc test, and for 16 the zone size around the amoxycillin disc was at least 5 $\mathrm{mm}$ smaller than that around the ampicillin disc. Clavulanate did not appear to enhance the activity of either ampicillin or amoxycillin. The MIC of ampicillin was about one-tenth that of amoxycillin (Table). Fifteen strains in this group appeared to be sensitive to ampicillin but resistant ot Augmentin. It is this type of resistance pattern which first suggested to us that Ent cloacae may be more sensitive to ampicillin than to amoxycillin. These 15 strains were distributed amongst eight different API biotypes. The other five strains destroyed amoxycillin, and were highly resistant to both ampicillin and amoxycillin (Table). They were also resistant to Augmentin and four were resistant to the ampicillin/ clavulanate disc.

\section{Discussion}

As the first four strains of Ent cloacae resistant to Augmentin but sensitive to ampicillin which we isolated came within a few weeks from patients whom we had treated with Augmentin, our first thought was that these may be atypical strains which had been selected by the use of Augmentin. However, subsequently-over a period of some 12 monthswe have only isolated one other such strain, from a patient given cotrimoxazole. This finding, and our observations that this type of Ent cloacae was common among unselected strains collected in the clinical laboratory before Augmentin came into use and that many API types are involved, suggests that such selection does not in fact take place.

Ent cloacae was not very common in our hospital: during 1981, Enterobacter spp accounted for only $1.7 \%$ of species isolated in significant numbers from urines, and only $1.3 \%$ of blood culture isolates. It is therefore much less common than in USA, where this genus is responsible for between 4 and $10 \%$ of bacteraemias. ${ }^{8} C$ freundii is in our experience rarer than Enterobacter spp, and again much less commonly found than in USA ${ }^{9}$. Finally, $S$ marcescens is virtually unknown in this hospital, although it has been reported from other centres in Great Britain.

The phenomenon of sensitivity to ampicillin coupled with resistance to Augmentin, which at first appeared to be an anomalous finding, is totally explained by the much greater activity of ampicillin (tenfold) than of amoxycillin against Ent cloacae (see Figure). The phenomenon was only noticed because we, like almost all other laboratories in this country, used "class" testing for determining sensitivity to amoxycillin. We now strongly recommend that where amoxycillin is in clinical use an amoxycillin disc be used for sensitivity testing, rather than ampicillin. This would also be helpful in the case of Haemophilus infuenzae, against which ampicillin is also more active than amoxycillin. ${ }^{10}$ In view of the existence of organisms such as Enterobacter spp which show a differential sensitivity, testing against both ampicillin and amoxycillin discs may be necessary.

We were surprised that so many strains of Enterobacter spp and $S$ marcescens were sensitive to ampicillin. These genera have been widely regarded as highly resistant to ampicillin, from the earliest days following the introduction of ampicillin. Before the advent of a rapid numerical taxonomic system-such as the API $20 \mathrm{E}$ system used in the present study-it was difficult to identify Enterobacter spp accurately, and indeed these strains used not to be separated from other biochemically related organisms, but were classified under the broad head- 
ing" "Klebsiella-Enterobacter-Serratia". However, in a major study by Toala $e t$ al,,$^{12}$ in which Ent cloacae and Ent aerogenes were fully identified, none of 199 strains of either species was inhibited by $100 \mu \mathrm{g} / \mathrm{ml}$ of ampicillin. It may be that the API $20 \mathrm{E}$ system identifies organisms as Enterobacter spp which would have been classified as other species by conventional means.

It is perhaps coincidence that the genera in which we were able to detect the phenomenon of ampicillin sensitivity but resistance to Augmentin and amoxycillin all produce a type $1 \beta$-lactamase. This type of enzyme is a cephalosporinase, which hydrolyses ampicillin very slowly. ${ }^{13-17}$ However, we have not found any data on the rate of hydrolysis of amoxycillin by this enzyme. Crump and Cansdale ${ }^{18}$ have suggested that clavulanate may induce type 1 $\beta$-lactamase, so that amoxycillin is destroyed more rapidly if it is present with clavulanate than if present alone. This type of mechanism has been shown to explain resistance of Ent cloacae to cefamandole in the presence of cefoxitin. The latter compound is an inducer of Enterobacter $\beta$-lactamase but inactive against this genus. The induced enzyme destroys cefamandole and thus causes resistance. ${ }^{19}$ Although we have not formally investigated the possibility that $\beta$-lactamase induction may occur, it seems unlikely to be of major importance in view of our finding that zone sizes around Augmentin discs were only rarely significantly smaller than those around amoxycillin alone. This effect was found for only three strains of the 63 tested (two $S$ marcescens and one $C$ freundii), and in no case did the presence of clavulanate decrease the activity of ampicillin.

Various other mechanisms have been called upon to explain the resistance of Ent cloacae to $\beta$-lactam antibiotics, ${ }^{2021}$ and it seems that much more work will be necessary before the exact resistance mechanism in this intriguing genus are fully understood.

We are grateful to $\operatorname{Dr} G \mathrm{~N}$ Rolinson and $\mathrm{Dr} R$ Sutherland of Beecham Research Laboratories for valuable discussions.

\section{References}

' Sutherland R, Croydon EAP, Rolinson GN. Amoxycillin: a new semi-synthetic penicillin. Br Med J 1972;iii:13-16.

${ }^{2}$ Hunter PA, Rolinson GN, Witting DA. Comparative activity of amoxycillin and ampicillin in an experimental bacterial infection in mice. Antimicrob Agents Chemother 1973;4:285-93.

${ }^{3}$ Comber KR, Osborne CD, Sutherland R. Comparative effects of amoxycillin and ampicillin in the treatment of experimental mouse infection. Antimicrob Agents Chemother 1975;7:179 85.

4 Barry AL, Thornsberry C. Susceptibility testing diffusion test procedures. In: Lennette EN, Balows A, Hausler WJ, Truant JP, eds. Manual of clinical microbiology (3rd ed). Washington: American Society for Microbiology, 1980:463-74.

s Brown D, Blowers R. Disc methods of sensitivity testing and other semiquantitative methods. In: Reeves DS, Phillips I, Williams JD, Wise R, eds. Laboratory methods in antimicrobial chemotherapy. Edinburgh: Churchill-Livingstone, 1978:8-30.

- Brumfitt W, Hamilton-Miller JMT, Dixson S, Gargan RA, Gooding A. Enterobacter resistant to amoxycillin/clavulanate. Lancet 1982;ii:768-9.

${ }^{7}$ Batchelor FR, Chain EB, Hardy TL, Mansford KRL, Rolinson GN. 6-aminopenicillanic acid III. Isolation and purification. Proc $R$ Soc Lond [ser B] 1961;154:498-508.

${ }^{8}$ John JF, Sharbaugh RJ, Bannister ER. Enterobacter cloacae: bacteraemia, epidemiology and antibiotic resistance. Rev Infect Dis 1982;4:13-28.

${ }^{9}$ Lipsky BA, Hook EW, Smith AA, Plorde JJ. Citrobacter infections in humans: experience at the Seattle Veterans Administration Medical Center and a review of the literature. Rev Infect Dis 1980;2:746-60.

${ }^{10}$ Kosmidis J, Williams JD, Andrews J, Goodall JAD, Geddes A. Amoxycillin-pharmacology, bacteriology and clinical studies. Br J Clin Pract 1972;26:341-6.

"Eickhoff TC, Steinhauer BW, Finland M. The KlebsiellaEnterobacter-Serratia division. Biochemical and serologic characteristics and susceptibility to antibiotics. Ann Intern Med 1966;65:1163-79.

12 Toala P, Lee YH, Wilcox C, Finland M. Susceptibility of Enterobacter cloacae and Enterobacter aerogenes to 19 antimicrobial agents in vitro. Am J Med Sci 1970;260:41-55.

${ }^{13}$ Hennessey TD, Richmond MH. The purification and some properties of a $\beta$-lactamase (cephalosporinase) synthesized by Enterobacter cloacae. Biochem J 1968;109:469-73.

${ }^{14}$ Farrar WE, Krause JM. Relationship between $\beta$-lactamase activity and resistance of Enterobacter to cephalothin. Infect Immun 1970;2:610-6.

1s Tsang JC, Sansing GA, Miller MA. Relationship of betalactamase activity to antimicrobial susceptibility in Serratia marcescens. Antimicrob Agents Chemother 1975;8:277-81.

${ }^{16}$ Sawai T, Mitsuhashi S, Yamagishi S. Drug resistance in enteric bacteria. XIV. Comparison of $\beta$-lactamase in Gram-negative rod bacteria resistant to $\alpha$-aminobenzylpenicillin. Jpn J Microbiol 1968;12:423-34.

${ }^{17}$ Minami S, Inoue M, Mitsuhashi S. Purification and properties of a cephalosporinase from Enterobacter cloacae. Antimicrob Agents Chemother 1980;18:853-5.

${ }^{18}$ Crump J. Cansdale S. Enterobacter resistant to amoxycillin/ clavulanate. Lancet 1982;ii:500.

19 Sanders CC, Sanders WE. Emergence of resistance to cefamandole: Possible role of cefoxitin-inducible beta-lactamases. Antimicrob Agents Chemother 1979;15:792-7.

${ }^{20}$ Then RL, Angehrn P. Trapping of nonhydrolyzable cephalosporins by cephalosporinases in Enterobacter cloacae and Pseudomonas aeruginosa as a possible resistance mechanism. Antimicrob Agents Chemother 1982;21:711-7.

${ }^{21}$ Sogaard P. Resistance types in Enterobacter cloacae. Acta Pathol Microbiol Scand [B] 1982;90:229-33.

Requests for reprints to: Professor W Brumfitt, Department of Medical Microbiology, The Royal Free Hospital, Pond Street, London NW3 2QG, England. 